\section{Stabilizing halide perovskite surfaces for solar cell operation with wide-bandgap lead oxysalts}

\author{
Shuang Yang ${ }^{1,2 *}$, Shangshang Chen ${ }^{1 *}$, Edoardo Mosconi $^{3}$, Yanjun Fang ${ }^{1,2}$, Xun Xiao', \\ Congcong Wang ${ }^{4}$, Yu Zhou', Zhenhua Yu', Jingjing Zhao', ${ }^{1,2}$, Yongli Gao", \\ Filippo De Angelis ${ }^{3,5,6}$, Jinsong Huang ${ }^{1,2} \dagger$
}

\begin{abstract}
We show that converting the surfaces of lead halide perovskite to water-insoluble lead (II) oxysalt through reaction with sulfate or phosphate ions can effectively stabilize the perovskite surface and bulk material. These capping lead oxysalt thin layers enhance the water resistance of the perovskite films by forming strong chemical bonds. The wide-bandgap lead oxysalt layers also reduce the defect density on the perovskite surfaces by passivating undercoordinated surface lead centers, which are defect-nucleating sites. Formation of the lead oxysalt layer increases the carrier recombination lifetime and boosts the efficiency of the solar cells to $21.1 \%$. Encapsulated devices stabilized by the lead oxysalt layers maintain $96.8 \%$ of their initial efficiency after operation at maximum power point under simulated air mass (AM) $1.5 \mathrm{G}$ irradiation for 1200 hours at $65^{\circ} \mathrm{C}$.
\end{abstract}

T he power conversion efficiency (PCE) of laboratory-scale metal halide organic perovskite solar cells (PSCs) has reached a certified value of $24.2 \%$, exceeding those of cells that are based on cadmium telluride (CdTe) and copper indium gallium selenide (CIGS) (1). However, PSCs face long-term instability issues under realistic operation conditions, which remains a critical hurdle to be overcome before their commercialization (2-4). Lead halide perovskites generally suffer from instability under stimuli of heat, oxygen, moisture, light irradiation, and electric field (4-6). Tailoring the composition, crystallinity, and internal material strain have been shown to substantially improve the materials' intrinsic stability under different stimuli, but their instability from moisture and oxygen is still an outstanding issue to be solved (7-10).

Perovskite degradation is generally initialized from the defect sites at surfaces and grain boundaries, which are more reactive toward water and oxygen (11-13). Many organic molecules and poly-

${ }^{1}$ Department of Applied Physical Sciences, University of North Carolina at Chapel Hill, Chapel Hill, NC 27599, USA.

${ }^{2}$ Department of Mechanical and Materials Engineering, University of Nebraska-Lincoln, Lincoln, NE 68588, USA.

${ }^{3}$ Computational Laboratory for Hybrid/Organic Photovoltaics (CLHYO), CNR-Istituto di Scienze e Tecnologie Molecolari (ISTM), Perugia, Italy. ${ }^{4}$ Department of Physics and Astronomy, University of Rochester, Rochester, NY 14627. USA. ${ }^{5}$ Department of Chemistry, Biology and Biotechnology, University of Perugia, Via Elce di Sotto 8, 06123 Perugia, Italy. ${ }^{6} \mathrm{D} 3$-Computation, Istituto Italiano di Tecnologia, Genova, Italy.

*These authors contributed equally to this work. †Corresponding author. E-mail: jhuang@unc.edu (J.H.) mers have been applied to chemically passivate the perovskite surface defects, and physical covering of some structural defects with hydrophobic organic materials can also enhance the stability of the perovskites (14-17). For example, $\mathrm{C}_{60}$ and its derivatives have been reported as passivation layers, enhancing device efficiency and reducing current hysteresis in PSCs (14). The surface under-coordinated $\mathrm{Pb}$ sites can be passivated by Lewis base electron donors, such as thiophene and pyridine, which saturate the lead coordination sphere (15). Zwitterions, bearing both negative and positive components, are shown to have a superior dual-passivation effect, leading to enhanced stability of unencapsulated perovskite films under ambient conditions (16).

Despite these initial successes, limitations exist in using these passivation agents as postsynthesis perovskite treatment for efficiency and stability enhancement. The secondary bonding between these passivation molecules and the perovskite surface is generally too weak to protect the material from attack from moisture and oxygen. Not all structural defects are electronic defects, so some structural defects that may initialize the film degradation might not be covered by passivation molecules.

The passivation strategies reported for halide perovskites are fundamentally different from those effectively used in silicon solar cells, in which passivation mainly involves primary chemical bonding. For silicon technology, surface structural defects are generally passivated by silicon oxide, silicon nitride, or aluminum oxide, which form strong covalent bonds to silicon. Oxides and nitrides are mechanically strong, compact, and chem- ically stable, which protect the underlying silicon from degradation. Wide-bandgap oxides and nitrides passivate the surface defects by eliminating silicon dangling bonds and thus enhance device efficiency.

We demonstrate a general passivation strategy for lead halide perovskites by forming a thin, compact inorganic lead oxysalt layer on the perovskite surface through in situ reaction with selected inorganic anions. The surface lead oxysalt layer formed strong chemical bonds with the perovskite and afforded much better resistance to many detrimental stimuli under ambient atmosphere and light irradiation. Its passivation effect enhanced the PSC efficiency.

The concept of metal halide perovskite surface passivation by using compact inorganic layers is illustrated in Fig. 1A. Inorganic layers for surface passivation of metal halide perovskite should have a large bandgap to reduce surface recombination velocity and be chemically stable in ambient air, especially under humid conditions. The lead salts that are insoluble in water are ideal candidates for perovskite surface stabilization. For example, the solubility of $\mathrm{PbSO}_{4}, \mathrm{~Pb}_{3}\left(\mathrm{PO}_{4}\right)_{2}$, and $\mathrm{PbCO}_{3}$ in water is merely $0.0443,0.00014$, and $0.0011 \mathrm{~g} \mathrm{liter}^{-1}$ at $20^{\circ} \mathrm{C}$, respectively (18). By contrast, $\mathrm{PbI}_{2}$ has a much higher solubility of $0.756 \mathrm{~g}$ liter $^{-1}$ in water.

Another requirement for the surface inorganic layers is that they must bond strongly to the perovskite surface so that the coating is mechanically strong. We formed $\mathrm{PbSO}_{4}$ and $\mathrm{Pb}_{3}\left(\mathrm{PO}_{4}\right)_{2}$ as surface inorganic layers through the facile chemical reaction of perovskite with $\mathrm{SO}_{4}{ }^{2-}$ - and $\mathrm{PO}_{4}{ }^{3-}$ containing salts dissolved in solvents that do not dissolve halide perovskites, such as isopropanol or toluene-in this case, $\left(\mathrm{C}_{8} \mathrm{H}_{17} \mathrm{NH}_{3}\right)_{2} \mathrm{SO}_{4}$ and $\left(\mathrm{C}_{8} \mathrm{H}_{17} \mathrm{NH}_{3}\right)_{3} \mathrm{PO}_{4}$. Triple-cation perovskite was used in our study with a chemical composition of $\mathrm{Cs}_{0.05} \mathrm{FA}_{0.81} \mathrm{MA}_{0.14} \mathrm{PbI}_{2.55} \mathrm{Br}_{0.45}$ (CsFAMA perovskite, where MA is methylammonium and FA is formamidinium). The oxyacid ions reacted with halide perovskite when perovskite films were dipped into $\left(\mathrm{C}_{8} \mathrm{H}_{17} \mathrm{NH}_{3}\right)_{2} \mathrm{SO}_{4}$ and $\left(\mathrm{C}_{8} \mathrm{H}_{17} \mathrm{NH}_{3}\right)_{3} \mathrm{PO}_{4}$ solutions for 30 to $\sim 60 \mathrm{~min}$ followed by thermal annealing, according to the following chemical equations

$$
\begin{gathered}
\mathrm{APbX}_{3}+\mathrm{SO}_{4}^{2-} \rightarrow \mathrm{A}^{+}+3 \mathrm{X}^{-}+\mathrm{PbSO}_{4} \\
3 \mathrm{APbX}_{3}+\underset{\mathrm{Pb}_{4}^{3-}\left(\mathrm{PO}_{4}\right)_{2}}{2 \mathrm{PO}^{+}+9 \mathrm{X}^{-}+}
\end{gathered}
$$

where $\mathrm{A}=\mathrm{MA}, \mathrm{FA}, \mathrm{Cs}$, or a mixture thereof, and $\mathrm{X}=\mathrm{Br}$, I, or mixed halide ions. This reaction yielded stable white-colored $\mathrm{PbSO}_{4}$ and $\mathrm{Pb}_{3}\left(\mathrm{PO}_{4}\right)_{2}$ films, as shown in the photographs and verified with x-ray diffraction patterns in figs. S1 and S2.

Using the same method, a thin lead sulfate layer can be generated on the surface of the CsFAMA perovskite films by reducing the reaction time. Because $\mathrm{PbSO}_{4}$ is electrically insulating, the reaction time and precursor concentration were carefully optimized to be $20 \mathrm{~s}$ and $4 \mathrm{mM}$, 
respectively, which yields a layer thickness of $<5 \mathrm{~nm}$ that can maintain efficient charge transport (fig. S3). Perovskite films or devices with lead sulfate layers, hereafter referred to as lead sulfate samples, were obtained through octylammonium sulfate treatment if not specified otherwise.

Scanning electron microscopy (SEM) and atomic force microscopy (AFM) images in figs. S4 and S5, A and B, show that the topography of the perovskite films was well maintained after the surface reaction, indicating a conformal coating of lead sulfate on the perovskites. Cross-sectional high-resolution transmission electron microscopy (HR-TEM) (Fig. 1B) further revealed a uniform and compact lead sulfate layer that was closely stacked between the fullerene $\left(\mathrm{C}_{60}\right)$ and perovskite films. The lead sulfate layer had a thickness of 3 to $4 \mathrm{~nm}$, whereas the $\mathrm{C}_{60}$ layer was crystalline. We used scanning TEM characterization to verify the homogeneity of the sulfate layer on the top of perovskite films. We tested various perovskite/ $\mathrm{C}_{60}$ interface regions (fig. S5C) and observed a thin but continuous lead sulfate layer both on the surface and at grain boundaries of the perovskite film, suggesting complete coverage of lead sulfate on the top surface of perovskite films.

Fourier transform infrared (FT-IR) transmission spectra of CsFAMA perovskite powders before and after surface treatment were collected to probe the interactions of sulfate ions with the perovskite. The tetrahedral symmetric sulfate ions typically present one broad peak at $\sim 1100 \mathrm{~cm}^{-1}$ of the triply degenerate $v 3$ band (19). The occurrence of $v 1$ band $\left(945 \mathrm{~cm}^{-1}\right)$ and split $v 3$ bands (950 to $\sim 1200 \mathrm{~cm}^{-1}$ ) (Fig. 1C) suggests a distortion of the tetrahedral structure of sulfate ions and formation of strong ionic bonds between $\mathrm{Pb}^{2+}$ and $\mathrm{SO}_{4}{ }^{2-}$ ions (20). The characteristic vibration peaks of the sulfate-treated sample were at the identical frequency (950 to $\sim 1200 \mathrm{~cm}^{-1}$ ) to those of pure $\mathrm{PbSO}_{4}$ powder (fig. S6), which implies the similar IR vibration behavior of the surface layer on the perovskite surface.

We performed x-ray photoemission spectroscopy (XPS) measurements to further characterize the perovskite surface composition after surface treatment. The $\mathrm{Pb} 4 \mathrm{f}$ spectrum exhibited two contributions, $4 \mathrm{f}_{7 / 2}$ and $4 \mathrm{f}_{5 / 2}$ (Fig. 1D), located at 138.6 and $143.5 \mathrm{eV}$ for the film without any treatment (control), respectively (21). The shift of the $\mathrm{Pb}$ peaks toward higher binding energies is evidence for the formation of stronger ionic bonding between $\mathrm{Pb}^{2+}$ and $\mathrm{SO}_{4}{ }^{2-}$ ions (22). The $\mathrm{S} / \mathrm{Pb}$ atom ratio was estimated to be 1.03 by the integrated area of corresponding peaks (Fig. 1E).

We performed first-principles density functional theory (DFT) computational analyses of the interaction between $\mathrm{MAPbI}_{3}$ and $\mathrm{PbSO}_{4}$ using a slab model of both materials and investigated the nature of their interface by means of ab initio Car-Parrinello Molecular Dynamics (CPMD). We initially considered a (001) MAI-terminated $\mathrm{MAPbI}_{3}$ surface covered by eight $\mathrm{PbSO}_{4}$ units arranged in a network similar to that of the perfect $\mathrm{PbSO}_{4}$ crystal but matching the lattice of $\mathrm{MAPbI}_{3}$. We evolved the system dynamics at $350 \mathrm{~K}$ for several picoseconds and observed the structural inter- face rearrangement. Starting from the ordered interface (Fig. 2A), we observed the progressive replacement of a surface MAI unit with a $\mathrm{PbSO}_{4}$ unit within a few picoseconds (Fig. 2, B to D), suggesting a stronger interaction between surface $\mathrm{Pb}$ atoms of $\mathrm{MAPbI}_{3}$ and $\mathrm{SO}_{4}{ }^{-}$units to form $\mathrm{PbSO}_{4}$. We further monitored whether a stronger interaction could exist between the $\mathrm{PbI}_{2}{ }^{-}$ terminated surface and $\mathrm{PbSO}_{4}$. We thus compared the energetics of interface interaction between $\mathrm{PbSO}_{4}$ and the two $\mathrm{MAPbI}_{3}$ surface terminations considering a more closely packed $\mathrm{PbSO}_{4}$ slab composed by $12 \mathrm{PbSO}_{4}$ units (Fig. 2, E and F, and table S1). These simulations also suggest that interaction between the $\mathrm{PbI}_{2}$-terminated surface and $\mathrm{PbSO}_{4}$ is significantly favored compared with MAI-terminated surface, and the theoretical studies are consistent with the experimental results that show a strong preferential interaction of sulfate anions with perovskite lead atoms. In all cases, bulk $\mathrm{PbSO}_{4}$ is more stable compared with the formation of a $\mathrm{MAPbI}_{3}$-surface $\mathrm{PbSO}_{4}$ thin film (table S1), which is consistent with the complete perovskite conversion to $\mathrm{PbSO}_{4}$ observed upon prolonged exposure to sulfate anions.

To further investigate the structure of the $\mathrm{PbSO}_{4}$ phase at the interface with $\mathrm{MAPbI}_{3}$, we built a larger model in which the $\mathrm{PbI}_{2}$-terminated $\mathrm{MAPbI}_{3}$ slab was interfaced with several layers of $\mathrm{PbSO}_{4}$ (fig. S7A). The O-S and O-Pb radial distribution functions showed no substantial changes from pure $\mathrm{PbSO}_{4}$ to the interface with $\mathrm{MAPbI}_{3}$ (fig. S7, B and C), which suggests the possibility for retention of a crystalline $\mathrm{PbSO}_{4}$ phase with good lattice matching. The experimental conditions may not favor the crystalline
A

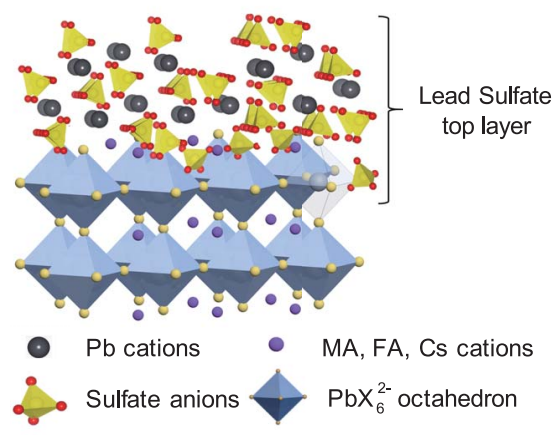

$\mathrm{PbSO}_{4}$ formation because of the speed of the reaction, but the amorphous $\mathrm{PbSO}_{4}$ layer was still effective in protecting perovskite surfaces.

Solar cells were fabricated with a p-i-n planar heterojunction configuration structured as indium tin oxide (ITO) glass substrate/poly(triaryl amine) (PTAA)/CsFAMA perovskite/ $\mathrm{C}_{60} / 2,9$-dimethyl-4, 7-diphenyl-1,10-phenanthroline (BCP)/Cu. The solar cell device with a lead sulfate surface layer delivered a short-circuit current density $\left(J_{\mathrm{SC}}\right)$ of $22.63 \mathrm{~mA} \mathrm{~cm}{ }^{-2}$, an open-circuit voltage $\left(V_{\mathrm{OC}}\right)$ of $1.16 \mathrm{~V}$, and a fill factor $(\mathrm{FF})$ of 0.804 , yielding a PCE of 21.1\% under a reverse scan rate of $0.1 \mathrm{~V} \mathrm{~s}^{-1}$ (Fig. 3A and table S2). No notable photocurrent hysteresis was observed under forward and reverse sweeps, which suggests good passivation by the lead sulfate layer. The $J_{\mathrm{SC}}$ value obtained from the current density-voltage $(J-V)$ characteristics was also verified by measuring the external quantum efficiency (EQE) obtained through integration of the spectral response (fig. S8). Additionally, the best device was further held at maximum power point (MPP) with a voltage of $0.99 \mathrm{~V}$ to track the stabilized power output. The photocurrent stabilized at $\sim 21.2 \mathrm{~mA} \mathrm{~cm}^{-2}$ (Fig. 3B), corresponding to a stabilized PCE of $21.0 \%$.

We measured $J-V$ characteristics of champion devices with different surface treatment under simulated sunlight of air mass (AM) $1.5 \mathrm{G}$ (Fig. 3C). The control device had a $J_{\mathrm{SC}}$ of 22.51 $\mathrm{mA} \mathrm{cm}{ }^{-2}$, a $V_{\mathrm{OC}}$ of $1.07 \mathrm{~V}$, and a $\mathrm{FF}$ of 0.796 , yielding a PCE of $19.16 \%$. The photovoltaic parameters of the devices with and without octylammonium sulfate treatment were analyzed, and the statistical distribution is shown in fig. S9. The

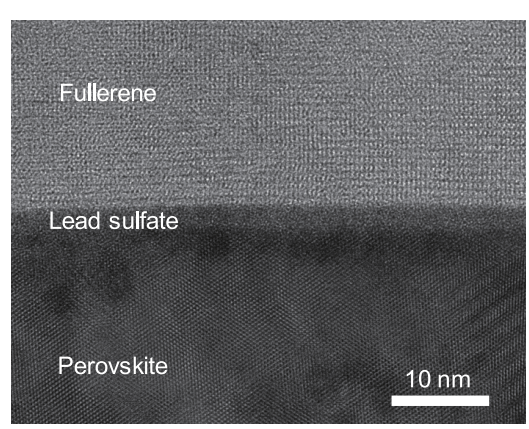

C

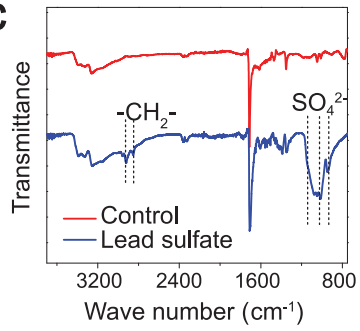

D

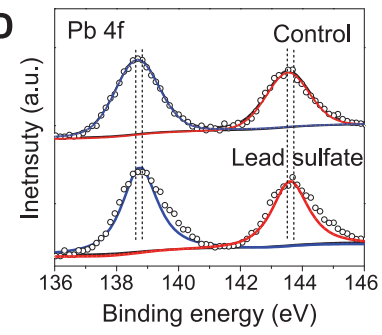

E

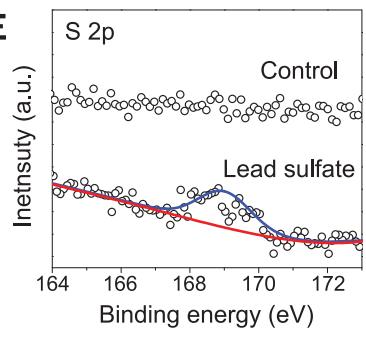

Fig. 1. Organohalide lead perovskite stabilized by a lead sulfate surface layer. (A) Schematic illustration of protection of perovskites through in situ formation of a lead sulfate top layer on the perovskite surface. (B) Cross-sectional HR-TEM image of the perovskite/lead sulfate/ $\mathrm{C}_{60}$ interface. (C) FT-IR measurement of perovskite powder with or without the lead sulfate layer. (D and $\mathbf{E})$ The XPS spectra of (D) Pb $4 f$ and (E) S 2p for the perovskite films deposited on ITO glass. 
A

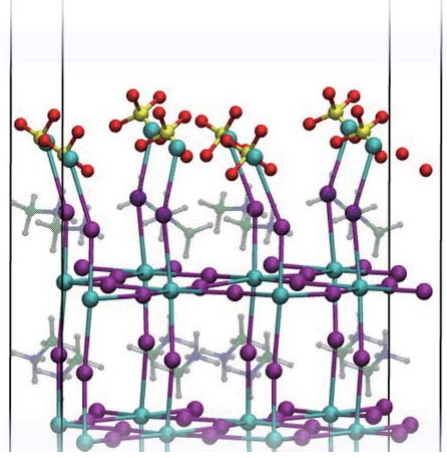

C

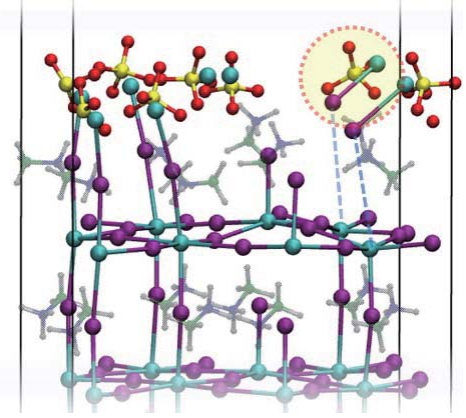

E

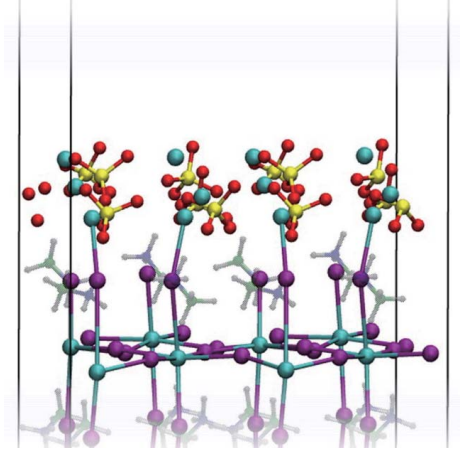

B

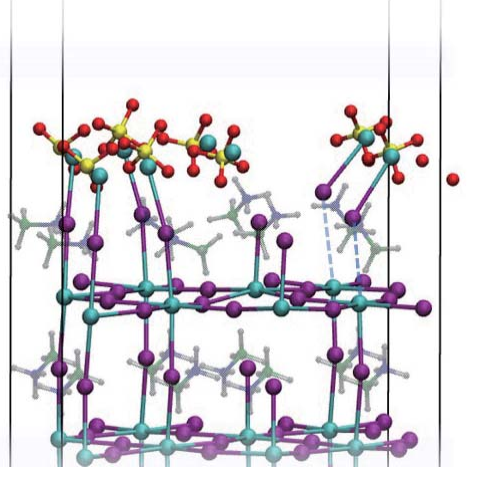

D

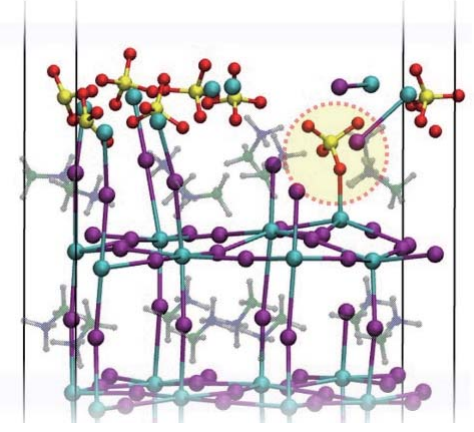

$\mathbf{F}$

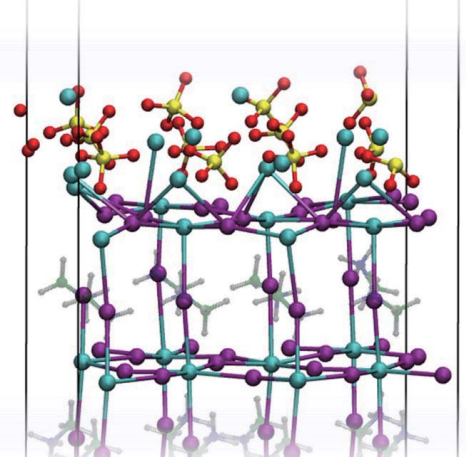

Fig. 2. Theoretical simulation. (A to F) Optimized geometries from the selected snapshots of the MAl-terminated $+8 \mathrm{PbSO}_{4}$ CPMD simulation. The $(\mathrm{A})$ initial geometry and the $[(\mathrm{B})$ and $(\mathrm{C})]$ optimized geometry after (B) 1.2 and (C) 3.6 ps are also shown. (D) The structure in which a MAI was exchanged with a $\mathrm{PbSO}_{4}$ from geometry $(\mathrm{C})$. (E) and $(\mathrm{F})$ show optimized geometries of (E) MAlterminated and $(\mathrm{F}) \mathrm{Pbl}_{2}$-terminated interface with a $\mathrm{PbSO}_{4}$ layer.

mean $V_{\mathrm{OC}}$ was 1.06 and $1.14 \mathrm{~V}$ for the control and sulfate-treated devices, respectively. Likewise, the mean PCE of the sulfate-treated devices reached $20.18 \pm 0.56 \%$. The enhanced $V_{\mathrm{OC}}$ and PCE as well as hysteresis-free behavior of the sulfate-treated devices together indicate the surface charge traps were passivated by the lead sulfate top layer.

To quantify how much cation passivates the perovskite surface, we fabricated solar cells with different cations and anions, and detailed statistical analysis on the device parameters are summarized in table S3. Octylammonium iodide treatment increased the mean $V_{\mathrm{OC}}$ of the cells from 1.06 to $1.08 \mathrm{~V}$, implying that there is a small but distinct contribution from alkylammonium cations to the passivation of perovskite surface. However, when anions were changed by replacing octylammonium iodide with octylammonium sulfate, the mean $V_{\mathrm{OC}}$ of the cells increased from 1.08 to $1.14 \mathrm{~V}$. Moreover, a high mean $V_{\mathrm{OC}}$ of $1.13 \mathrm{~V}$ could be maintained if methylammonium sulfate was used instead of long-chain alkylammonium cations, implying that sulfate anions dominated in surface passivation. We infer that the perovskite films are mostly covered by the lead sulfate layer after treatment because of the preferential reaction between $\mathrm{Pb}$ cations and sulfate anions, as indicated by the results of DFT simulations, so octylammonium cations have less interaction with the perovskite surface. We further demonstrated the universality of this synthetic strategy of using phosphate ions to form a $\mathrm{Pb}_{3}\left(\mathrm{PO}_{4}\right)_{2}$ surface layer and examined the device performance. One solar cell device based on phosphated CsFAMA perovskite exhibited a $J_{\mathrm{SC}}$ of $22.54 \mathrm{~mA} \mathrm{~cm}^{-2}, V_{\mathrm{OC}}$ of $1.14 \mathrm{~V}$, and $\mathrm{FF}$ of 0.813 , yielding a PCE of $20.87 \%$ without hysteresis (fig. S10 and table S4).

Trap density of states (tDOS) of the control and sulfate-treated devices was measured with thermal admittance spectroscopy. A device with sulfate top layers had a lower tDOS almost over the whole trap depth region (Fig. 3D). The density of shallower trap states ( 0.35 to $0.42 \mathrm{eV}$ ) of the sulfate-treated device was only about $10 \%$ that of the control device. Our previous characterizations have revealed that the shallow traps mainly locate at the grain boundaries (23). Thus, sulfate ions can effectively reach the grain boundaries during the treatment and passivate them, subsequently increasing the device $V_{\mathrm{OC}}$. The time-resolved photoluminescence (TRPL) spectra of the control CsFAMA perovskite film show a biexponential decay with a fast and a slow component (Fig. 3E). The surface layer mainly affected the fast PL decay process. The fast-component of the recombination process from the control and sulfate-treated films were fitted to be 3.2 and $24.7 \mathrm{~ns}$, respectively, which is consistent with the enhanced steady-state PL results (Fig. 3F). The enhanced PL intensity and PL decay lifetime illustrate that the nonradiative recombination of photo-generated carriers in perovskite films is reduced by the formation of lead sulfate surface layers $(24,25)$.

Devices were then irradiated under AM 1.5 G simulated illumination, and weak laser pulses $(337 \mathrm{~nm}, 4 \mathrm{~ns})$ were used to modulate $V_{\mathrm{OC}}$ to measure the decay of transient photovoltage signals. As seen in Fig. $3 \mathrm{G}$ and fig. S11, the chargerecombination lifetime under 1 Sun illumination was increased from $0.27 \mu$ s for the control device to 0.58 and $0.47 \mu$ s for sulfate- and phosphatetreated devices, respectively. The lower charge trap density and longer carrier recombination lifetime of samples with lead sulfate top layers indicate that the reaction of sulfate and phosphate ions with defective perovskite surface reduces the surface defect density, in addition to the charge compensation effect of these ions. The widebandgap lead oxysalt layer should also contribute to the reduced surface charge recombination rate, similar to the passivation of silicon by oxides (26).

We performed long-term stability tests of encapsulated CsFAMA perovskite devices under a plasma lamp with light intensity equivalent to AM 1.5 G (fig. S12), without ultraviolet filter in air (relative humidity $\sim 60 \pm 10 \%$ ). All devices were loaded with a resistance so that they worked at MPP at the beginning of the tests. The $J$ - $V$ curves were automatically recorded with a reverse scan rate of $0.1 \mathrm{~V} \mathrm{~s}^{-1}$ every 6 hours. We frequently checked the stabilized efficiency during the test and did not find obvious differences between the stabilized efficiency and that from $J-V$ scanning. Most importantly, instead of monitoring device stability at a reduced temperature of 20 to $25^{\circ} \mathrm{C}$ that may cause the overestimation of solar cell 
stability, we conducted the stability testing at real operation temperature and used a light source with a substantial ultraviolet component. The temperature of the devices under illumination was measured to be $\sim 65^{\circ} \mathrm{C}$, which is typical of the thermal heating effect of light. The PCE of the encapsulated control device degraded rapidly from 18.2 to $8.54 \%$ after testing for 474 hours (Fig. $3 \mathrm{H}$ ). For the devices with the lead sulfate layer, the PCE slightly improved during the first 120 hours of testing and then degraded linearly with both reduced $J_{\mathrm{SC}}$ and FF over time. After 1200 hours of testing, the efficiency slightly dropped to $96.8 \%$ of the initial value. This CsFAMA perovskite device is one of the most stable reported when tested at MPP conditions (table S5).
In ambient conditions, hydration and oxidization of perovskites are among main paths for the degradation of perovskite films $(13,27)$. We studied the water resistance of perovskites with lead sulfate surface layers on $\mathrm{MAPbI}_{3}$ single crystals. $\mathrm{MAPbI}_{3}$ crystals were treated with the sulfate precursor solution followed by thermal annealing at $100^{\circ} \mathrm{C}$ for $10 \mathrm{~min}$ (supplementary materials). When dipped in water, the control $\mathrm{MAPbI}_{3}$ crystal without any treatment quickly turned yellow within $10 \mathrm{~s}$ because of fast decomposition of $\mathrm{MAPbI}_{3}$ with water, forming $\mathrm{PbI}_{2}$ or other hydrates, whereas the sample with a sulfate layer remained black after dipping in water for $>60 \mathrm{~s}$ (Fig. $4 \mathrm{~A}$ and movie S1). The much later appearance of yellow $\mathrm{PbI}_{2}$ from perovskite single crystals showed that the lead sulfate top layer was compact enough to slow down the permeation of water into perovskite.

We tested the water resistivity of the lead sulfate layer by dripping water on top of the devices with and without sulfate treatment. A device with the lead sulfate layer remained black for 3 min after dripping water, whereas the control device quickly decomposed to yellow-colored $\mathrm{PbI}_{2}$ (fig. S13). We also tested the protection effect of lead sulfate layer on polycrystalline films that had the same stacking structure of the devices but without metal electrodes. The perovskite film with lead sulfate layer was sandwiched between PTAA and phenyl C61 butyric acid methyl ester (PCBM) layers. We recorded the
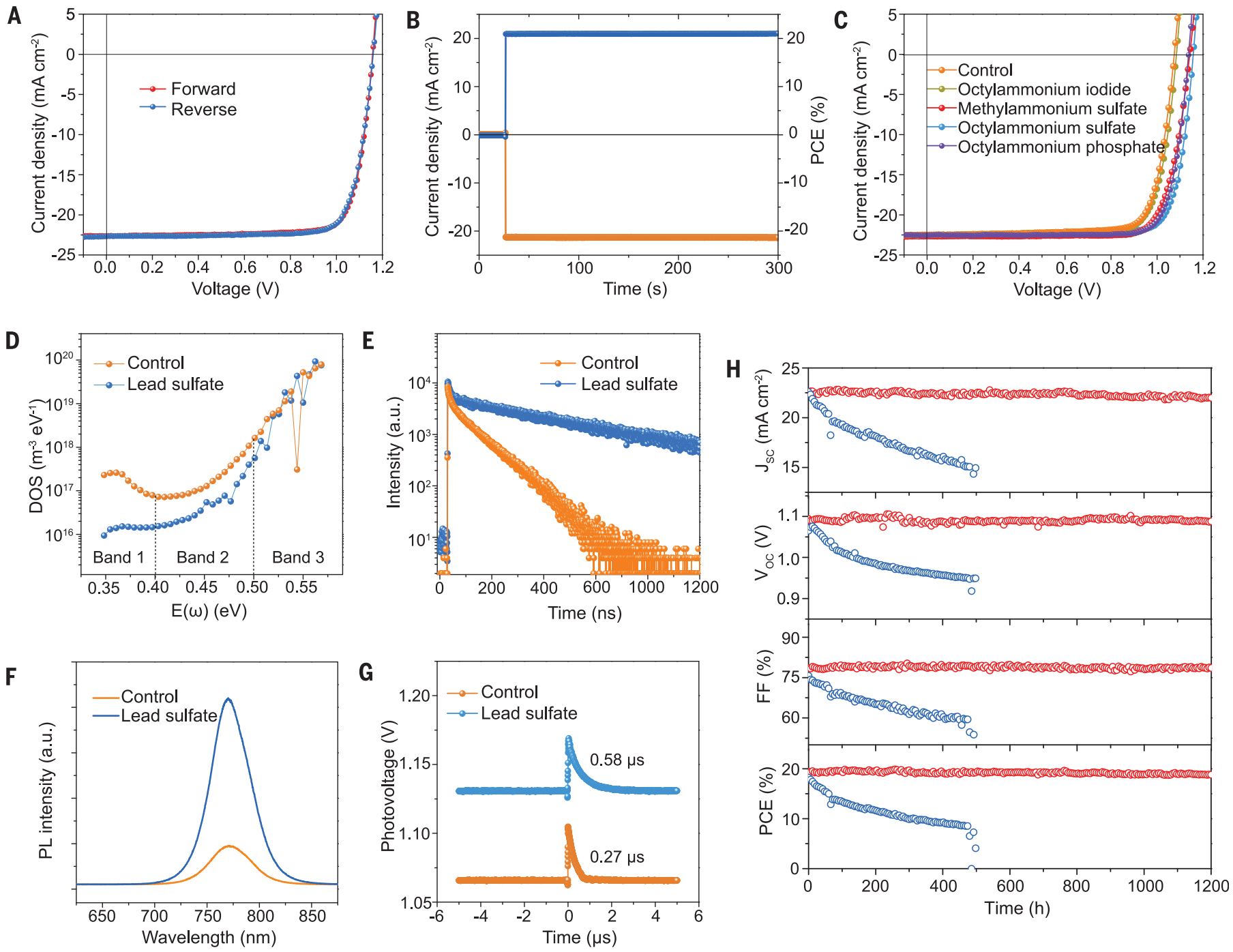

Fig. 3. Photovoltaic performance, passivation effects, and long-term stability studies of sulfate-treated devices. (A) $J-V$ curves of the champion sulfate-treated device measured in both reverse (blue) and forward (red) scanning directions. (B) Steady-state measurement of the photocurrent (orange) and PCE (blue) of the champion device with a lead sulfate layer held at MPP voltage of $0.99 \mathrm{~V}$. (C) J-V curves of perovskite solar cells based on perovskite films treated with different precursor solution. (D) tDOS obtained through thermal admittance spectroscopy for the devices with (blue) or without (orange) lead sulfate top layers. The vertical dashed lines define trap bands with different trap energy depths: shallow trap states (band 1, 0.35 to $0.40 \mathrm{eV}$ ) and deep trap states (band 2 and band $3,>0.4 \mathrm{eV}$ ). (E) Steady-state PL and (F) time-resolved PL of the perovskite films with and without lead sulfate layers. (G) TPV decay curves of the photovoltaic devices with and without lead sulfate surface layers. (H) Stability test of encapsulated solar cell devices based on control (blue) and sulfate-treated (red) CsFAMA perovskite active layers. 
A
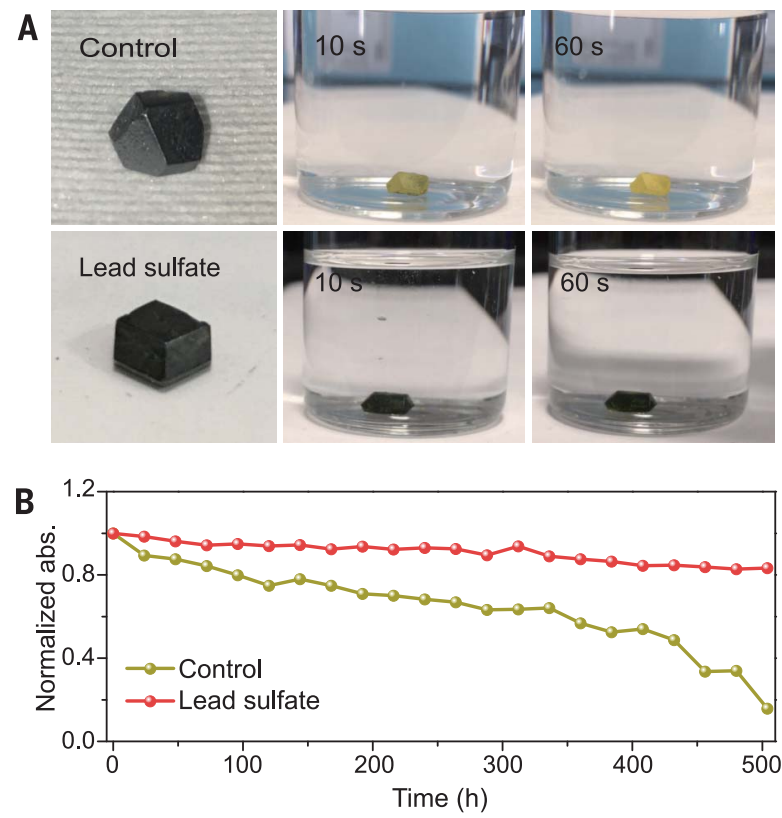

C

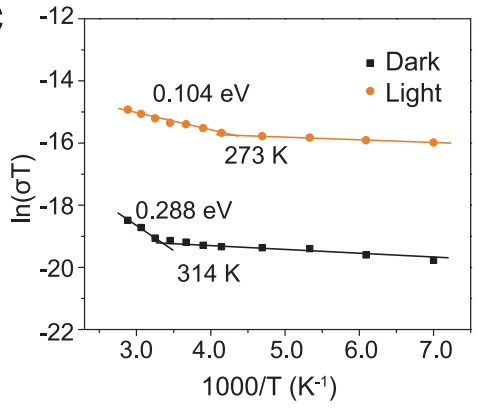

E

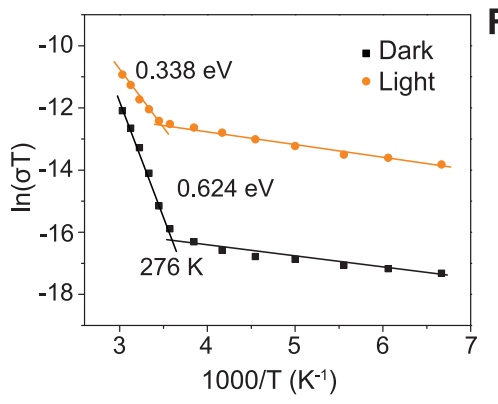

D

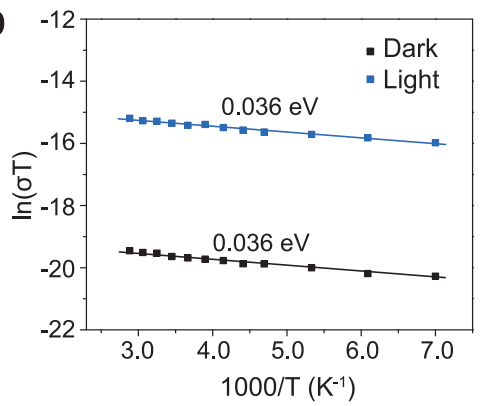

$\mathbf{F}$

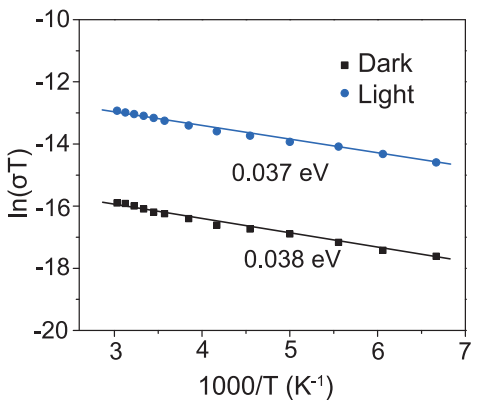

Fig. 4. Enhanced water resistance and suppressed ion migration by lead sulfate surface layer. (A) $\mathrm{MAPbl}_{3}$ single crystals without and with lead sulfate top layers dipped into water at different time intervals. (B) Normalized absorbance decay at $740 \mathrm{~nm}$ for perovskite films sandwiched between PTAA and PCBM layers under simulated AM 1.5 G irradiation (100 $\left.\mathrm{mW} \mathrm{cm}^{-2}\right)$ in ambient air. (C to F) Temperature-dependent conductivity of the PTAA/CsFAMA perovskite/PCBM films (C) without and (D) with methylammonium sulfate treatment, and $\mathrm{MAPbl}_{3}$ single crystal $(E)$ without and $(F)$ with methylammonium sulfate treatment. The light intensity is $10 \mathrm{~mW} \mathrm{~cm}^{-2}$ for conductivity measurements. absorbance changes at $740 \mathrm{~nm}$ during the stability test to quantify the material degradation. The control sample lost $84.2 \%$ of its absorbance after illumination for 504 hours, whereas the sample with a lead sulfate layer only lost $16.8 \%$ (Fig. 4B and fig. S14).

Ammonium cations have also been reported to enhance the stability of perovskites (28). We tested another set of perovskite thin films in air under illumination without any charge transport layers to study which species-specifically, ammonium or sulfate ions-play the dominating role in stabilizing perovskite films. The CsFAMA perovskite film treated with octylammonium iodide fully bleached after testing for 4 days, only slightly preventing the decomposition of the perovskite film as compared with the control sample without any treatment (fig. S15), but perovskite films with sulfate treatment remained black after 4 days. About 56 and $64 \%$ of the films' absorbance at $740 \mathrm{~nm}$ remained for films treated with methylammonium sulfate and octylammonium sulfate, respectively. The result verified that the stabilization was mainly derived from sulfate rather than ammonium ions.

Mass transport of ions is another critical issue that limits the stability of the encapsulated perovskite devices. Ion migration is significantly enhanced under illumination (29), which may change the composition and morphology of perovskite films by forming pinholes in addition to causing the degradation of charge transport layers and electrodes $(30)$. We also showed that ion migration is much easier at extended defects such as film surface and grain boundaries (31). We expect that the formation of a layer of lead oxysalt with strong ionic chemical bonding should stabilize the perovskite surface and suppress the ion migration through it. We measured with temperature-dependent electrical conductivity the activation energy $\left(E_{\mathrm{a}}\right)$ for ion migration of perovskite films. Lateral structure devices were fabricated by means of thermal evaporation of two Au electrodes on PTAA/ perovskite/PCBM films. The activation energy can be extracted from the Nernst-Einstein relation: $\sigma(T)=$ $\left(\sigma_{0} / T\right) \exp \left(-E_{\mathrm{a}} / k_{\mathrm{B}} T\right)$, where $k_{\mathrm{B}}$ is the Boltzmann constant, $\sigma_{0}$ is a constant, and $T$ is temperature. The applied electric field was fixed at $0.4 \mathrm{~V} / \mu \mathrm{m}$, which was near the operational electric field in solar cell devices. For the CsFAMA perovskite films, ionic conductivity began to dominate the total conductivity, with an $E_{\mathrm{a}}$ of $0.288 \mathrm{eV}$ when the temperature was increased to $314 \mathrm{~K}$ in the dark (Fig. 4C). When illuminated at 0.1 sunlight intensity, the transition temperature was reduced to $273 \mathrm{~K}$, accompanied with a lower $E_{\mathrm{a}}$ of $0.104 \mathrm{eV}$. This observation agrees well with our previous results that light would facilitate the ion migration (29).

For the sulfate-treated perovskite film, we did not observe such a transition from electronic to ionic conductivity when the temperature was increased to $330 \mathrm{~K}$ both in the dark and under illumination (Fig. 4D). A constant slope was obtained with an $E_{\mathrm{a}}$ of $0.036 \mathrm{eV}$, which we ascribed to electronic conduction. We also measured $E_{\mathrm{a}}$ of individual $\mathrm{MAPbI}_{3}$ single crystals to isolate the influence of untreated grain boundaries in polycrystalline films, which act as the main channel for ion migration (31). Similar phenomena were observed for the single crystals (Fig. 4, E to $F$ ). We thus conclude that ion migration is efficiently suppressed by the lead sulfate top layer on the surface of perovskite, possibly because surface defects such as vacancies were immobilized by the strongly bonded lead sulfate layer, which also explains the restrained morphological variation of perovskite films with the presence of the lead sulfate layer (fig. S16). Generally, ion migration through grain boundaries is much slower than at the surface, so the exclusion of ion migration at a film surface should also indicate the absence of ion migration through sulfated grain boundaries.

\section{REFERENCES AND NOTES}

1. National Renewable Energy Laboratory, Best research-cell efficiencies chart (2019); www.nrel.gov/pv/assets/pdfs/bestresearch-cell-efficiencies.20190703.pdf.

2. M. Grätzel, Nat. Mater. 13, 838-842 (2014).

3. S. Yang et al., Nat. Energy 1, 15016 (2016).

4. T. Leiitens et al., Adv. Energy Mater. 5, 1500963 (2015).

5. W. Nie et al., Nat. Commun. 7, 11574 (2016).

6. K. Domanski et al., Energy Environ. Sci. 10, 604-613 (2017).

7. R. J. Sutton et al., Adv. Energy Mater. 6, 1502458 (2016).

8. J. W. Lee et al., Adv. Energy Mater. 5, 1501310 (2015).

9. C. Yi et al., Energy Environ. Sci. 9, 656-662 (2016).

10. J. Zhao et al., Sci. Adv. 3, 05616 (2017).

11. Q. Wang et al., Energy Environ. Sci. 10, 516-522 (2017).

12. Z. Fan et al., Joule 1, 548-562 (2017).

13. N. Aristidou et al., Nat. Commun. 8, 15218 (2017)

14. J. Xu et al., Nat. Commun. 6, 7081 (2015).

15. N. K. Noel et al., ACS Nano 8, 9815-9821 (2014).

16. X. Zheng et al., Nat. Energy 2, 17102 (2017).

17. V. K. Ravi, R. A. Scheidt, A. Nag, M. Kuno, P. V. Kamat, ACS Energy Lett. 3, 1049-1055 (2018).

18. H. Stephen, T. Stephen, Solubilities of Inorganic and Organic Compounds (Macmillan, 1963), vol. 1. 
19. D. Peak, R. G. Ford, D. L. Sparks, J. Colloid Interface Sci. 218, 289-299 (1999).

20. E. Elzinga, D. Peak, D. Sparks, Geochim. Cosmochim. Acta 65 2219-2230 (2001).

21. J. H. Yun et al., J. Mater. Chem. A 3, 22176-22182 (2015).

22. S. J. 0. Hardman et al., Phys. Chem. Chem. Phys. 13, 20275-20283 (2011).

23. Y. Shao, Z. Xiao, C. Bi, Y. Yuan, J. Huang, Nat. Commun. 5 , 5784 (2014).

24. D. W. deQuilettes et al., Science 348, 683-686 (2015).

25. W. S. Yang et al., Science 356, 1376-1379 (2017).

26. L. E. Black, New Perspectives on Surface Passivation: Understanding the $\mathrm{Si}_{-} \mathrm{Al}_{2} \mathrm{O}_{3}$ Interface (Springer, 2016).

27. M. Wang, Sci. Bull. (Beijing) 62, 249-255 (2017).

28. A. Rajagopal, R. J. Stoddard, S. B. Jo, H. W. Hillhouse, A. K.-Y. Jen, Nano Lett. 18, 3985-3993 (2018)

29. J. Xing et al., Phys. Chem. Chem. Phys. 18, 30484-30490 (2016).

30. Y. Yuan, J. Huang, Acc. Chem. Res. 49, 286-293 (2016)

31. Y. Shao et al., Energy Environ. Sci. 9, 1752-1759 (2016).

\section{ACKNOWLEDGMENTS}

Funding: J.H. acknowledges supports from Office of Naval Research under award N00014-17-1-2727 and Air Force Office of Scientific Research (AFOSR) (grant A9550-16-1-0299). Y.G. acknowledges supports from National Science Foundation (NSF) CBET-1437656. F.D.A. and E.M. acknowledge the Ministero Istruzione dell'Università e della Ricerca (MIUR), the University of Perugia (grant AMIS), and ESPResSo Project (Horizon 2020, grant 764047). This work was performed in part at the Analytical

Instrumentation Facility (AIF) at North Carolina State University, which is supported by the state of North Carolina and NSF (award

ECCS-1542015). Author contributions: J.H. conceived the idea and supervised the research of this work. S.Y. and S.C. fabricated and characterized the perovskite films and solar cells. E.M. and

F.D.A. did the theoretical simulation. Y.F. conducted the

temperature-dependent conductivity measurement. Y.Z. and X.X. carried out the PL and TRPL characterizations. Z.Y. and J.Z. did the encapsulation and stability tests of perovskite devices.

C.W. and Y.G. conducted the XPS tests. J.H., S.Y., and S.C. wrote the manuscript, and all the authors reviewed the manuscript.
Competing interests: J.H. and S.Y. are inventors on U.S. patent application $(62 / 798,671)$ submitted by the University of Nebraska-Lincoln and University of North Carolina at Chapel Hill that covers conversion of halide perovskite surfaces to insoluble, wide-bandgap lead oxysalts for enhanced solar cell stability.

Data and materials availability: All data are available in the manuscript or the supplementary materials.

\section{SUPPLEMENTARY MATERIALS}

science.sciencemag.org/content/365/6452/473/suppl/DC1 Materials and Methods

Figs. S1 to S16

Tables S1 to S5

References (32, 33)

Movie S1

13 March 2019; resubmitted 29 April 2019

Accepted 3 July 2019

10.1126/science.aax3294 